\title{
Special issue WGP: “Assembly, Handling and Industrial Robotics"
}

\author{
Bernd Kuhlenkötter · Jörg Franke • \\ Thorsten Schüppstuhl · Alexander Verl
}

Published online: 8 October 2014

(c) German Academic Society for Production Engineering (WGP) 2014

This special issue contains a number of publications in the area of production research. The intention is to give innovative examples of the work of research institutes and to show some of recent developments from the interdisciplinary field of automation and robotics.

Nowadays automation is no longer only technology for producing big numbers of identical products. The challenges of the market have changed more and more: requests for more customer specific products are going along with a reduction of the time-to-market as well as a high production quality and a zero fault tolerance. And robots are no longer only applied for their former typical production purposes: robots-or other applicable kinematical structures-are also serving in repair and machining domains more and more.

These demands are the basis and the challenge for the research area, in universities and institutes as well as in R\&D departments of companies. And the diversity of demands results in research in multiple areas, ranging from gipping technologies up to new structures for activating robot links, with software based methods for planning, simulating and setting up the systems.

But it is not only basic research that has to be doneapplying the results to implementations in industrial systems is the next step. Therefore this issue presents achievements that are ready for the market, with developments being motivated,

B. Kuhlenkötter $(\bowtie) \cdot$ J. Franke

Dortmund, Germany

e-mail: bernd.kuhlenkoetter@tu-dortmund.de

T. Schüppstuhl

Hamburg, Germany

A. Verl

Munich, Germany driven, evaluated and confirmed by industrial partnersresults representing the practical impact that will bridge the gap between research and successful industrial applications.

The aim of this issue is to present innovative approaches and realizations on the overall way from the product idea via construction and engineering processes to an appropriate production system.

This issue was initiated by the "Academic Society for Assembly, Handling and Industrial Robotic" (Wissenschaftliche Gesellschaft für Montage, Handhabung und Industrierobotik (MHI)). The "Academic Society for Assembly, Handling and Industrial Robotic" is an organization of university professors being heads of institutes or chair holders. The members do basic research and application engineering in assembly, handling and robotics industry. MHI has currently 18 members representing approximately 1.000 scientists in their institutes and chairs. The society works in close cooperation with the German Engineering Federation (VDMA). In addition, MHI is supported by an industrial advisory board, consisting of about 12 members from industrial companies, representing technology supplier as well as users in the areas of assembly, handling and industrial robotics.

This is also the place to thank all the people that were involved in initiating this issue, contributing by papers as well as serving as reviewers and editorial committee or were part of the overall process to bring this special issue into a printed booklet.

Bernd Kuhlenkötter, Jörg Franke, Thorsten Schüppstuhl, Alexander Verl (Board of Directors of MHI-Academic Society for Assembly, Handling and Industrial Robotics/ Wissenschaftliche Gesellschaft für Montage, Handhabung und Industrierobotik)

Marion Merklein (Editor-in-Chief) 